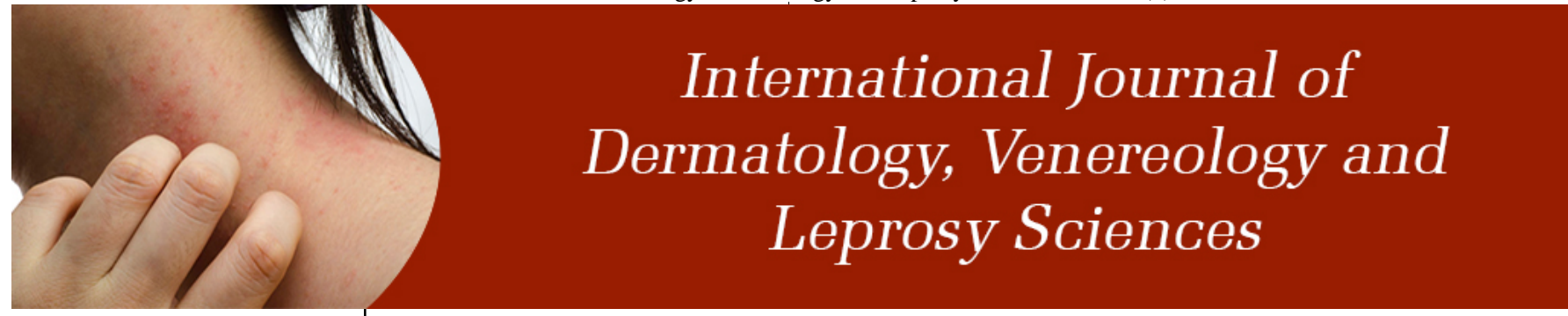

E-ISSN: 2664-942X P-ISSN: 2664-9411 www.dermatologypaper.com Derma 2021; 4(2): 83-88 Received: 25-07-2021 Accepted: 27-09-202

Dr. Tripti Upadhyay Junior Resident, Department of Dermatology, Subharti Medical College, Meerut, Uttar Pradesh, India

Dr. Arvind Krishna

Head of the Department, Department of Dermatology, Subharti Medical College, Meerut, Uttar Pradesh, India

Dr. Robin Chugh Associate Professor, Department of Dermatology, Subharti Medical College, Meerut, Uttar Pradesh, India

Dr. Abhinav David Senior Resident, Department of Dermatology, Subharti Medical College, Meerut, Uttar Pradesh, India
Corresponding Author: Dr. Tripti Upadhyay Junior Resident, Department of Dermatology, Subharti Medical College, Meerut, Uttar Pradesh, India

\section{An observational study describing dermoscopic features of common macular facial hyperpigmented skin lesions}

\author{
Dr. Tripti Upadhyay, Dr. Arvind Krishna, Dr. Robin Chugh and Dr. \\ Abhinav David
}

DOI: https://doi.org/10.33545/26649411.2021.v4.i2a.100

\begin{abstract}
Background: With this study we wanted to describe dermoscopic features in a variety of hyperpigmented lesions on face in Indian skin type with the help of Dino Capture 2.0 handheld dermoscope.

Materials and Methods: An observational analytical study was conducted on 150 clinically diagnosed patients of macular facial hyper-pigmented skin lesions attending the out-patient department of Dermatology, Venereology and Leprosy of Chhatrapati Shivaji Subharti Hospital, Meerut, for 18 months after approval by the university. This study was a prospective comparative study done in both sexes, age group $>18$ years of clinically diagnosed macular facial hyper-pigmented skin lesions among 150 clinically diagnosed subjects.

Results: In our study, mean age of the patients was $31.19 \pm 11.10$ years with minimum age of 18 years and maximum 74 years. In our study, more than two third of the patients $(72.0 \%)$ were females, while $28.0 \%$ were males. Males to females ratio was $7: 18$. The most common dermoscopic pattern in melasma were incomplete reticular pigmentation $(46.5 \%)$ and telangiectasia $(29.8 \%)$ followed by complete reticular pattern (18.4\%), granular pattern (14.9\%), and non-specific features (14.0\%).

Conclusion: Dermoscopy is useful in diagnosis, prognosis, and follow up of disorders of hyperpigmentation. With regular practice and attention to detail towards dermoscopic findings, it can be a useful tool in dermatology practice in general and for disorders of hyperpigmentation.
\end{abstract}

Keywords: Dermoscopy, facial hyperpigmentation, melasma

\section{Introduction}

Normal skin color is determined by a number of chromophores, the most important of which is melanin. Besides melanin, other chromophores that contribute significantly to skin color include hemoglobin (in both the oxygenated and reduced state) and carotenoids. Racial and ethnic differences in skin color are related to the number, size, shape, distribution and degradation of melanin-laden organelles called melanosomes. These are produced by melanocytes and are transferred to the surrounding epidermal keratinocytes. Hypermelanosis is an increased amount of melanin in the skin. This excess may be confined to the epidermis, when the skin appears browner than normal, or it may be present in the dermis, producing a slate gray or blue appearance. Hypomelanosis, where there is a lack of pigment in the skin, which therefore appears white or lighter than the normal color. Amelananosis is the term applied when there is a total lack of melanin in the skin ${ }^{[11]}$.

Melanin is not a single compound; rather, it is a mixture of biopolymers synthesized by melanocytes located in the basal layer of the epidermis. Based on their chemical composition, melanins are broadly classified into two types: eumelanin and pheomelanin ${ }^{[2]}$. Multiple studies have reported that individuals with darker skin have higher total melanin content, and a higher amount of eumelanin than lighter-skinned individuals. 
Furthermore, studies on cultured human melanocytes have demonstrated that melanocytes derived from darker skin have higher total melanin and eumelanin contents, and a higher ratio of eumelanin to pheomelanin, than those derived from lighter skin ${ }^{[3,4]}$. Pheomelanin differs from eumelanin in its biological behaviour, importantly in the ability of pheomelanin to activate oxygen resulting in the formation of the superoxide radical anion ${ }^{[5]}$.

Colors in dermoscopy depend on the location of the melanin in the skin (black, stratum corneum; brown, epidermis; grey, upper dermis; steel blue, medium dermis). Other colors seen in dermoscopy are red (inflammation, vascularity), white (depigmentation, scarring), yellow (hyperkeratosis, sebaceous material), orange (serum) and jet black (congealed blood) ${ }^{[2]}$.

Discoloration of the skin may also be caused by deposition of exogenous substances such as drugs, drug complexes (e.g. with melanin or iron) or heavy metals within the dermis. Increased pigmentation is also a well-known feature of many endocrine as well as other systemic disorders including Addison's disease, during pregnancy and OCP uses, renal failure, primary biliary cirrhosis and anemia due to vitamin B12 and folate deficiency. Hyperpigmentation involving predominantly the face and the neck is relatively common and often presents a complex diagnostic problem and trouble the patient about their appearance.

The common hyperpigmented macular facial skin lesions include melasma, lichen planus pigmentosus, periorbital hyperpigmentation, flat seborrheic keratosis, nevus of Ota, Hori's nevus, ochronosis, lentigines, freckles, post inflammatory hyperpigmentation, lentigo senilis, Riehl's melanosis, etc. ${ }^{[2]}$

Melasma is a common dyschromia located on sun-exposed areas, mainly on the face and occasionally on the neck and forearms. Melasma is due to the increased focal epidermal activity of melanocytes producing melanin, and microscopically there is increased melanin in epidermal keratinocytes and/or dermal macrophages [6]. It occurs mostly in women, and is clinically characterized by patterned, symmetric, brown to grayish-brown, hyperpigmented patches of the face in malar and centrofacial areas ${ }^{[6]}$. However, in India, in one reported series, one out of every five patients was male ${ }^{[7]}$.

Currently, the diagnoses of hyper pigmentary conditions are made by clinical examination. However, the clinical features are inappropriate and subjective in many patients. The use of biopsy for a confirmatory diagnosis is avoided by its invasive nature. Any clinical modality that helps to provide an accurate diagnosis without being invasive in nature is of great importance and hence the role of dermoscopy.

Dermoscopy (also known as epiluminescence microscopy, dermatoscopy and amplified surface microscopy) is an in vivo non-invasive method for observation of pigmented skin lesions. Apart from dermoscopy, digital dermoscopy and video dermoscopy are used to monitor the patients, as well as tele dermoscopy, which provides new opportunities in this field ${ }^{[2]}$.

In a tropical country like India, fair skin is prized above a healthy, even-toned facial skin, with facial pigmentation translating into a significant psychosocial problem and a frequent cause of dermatologic consultation. In the Indian context, common causes of facial melanosis include melasma, Riehl's Melanosis, Lichen planus pigmentosus, facial acanthosis nigricans, pigmentary demarcation lines, post-inflammatory hyperpigmentation ${ }^{[8]}$.

With this study we wanted to describe dermoscopic features in a variety of hyperpigmented lesions on face in Indian skin type with the help of DinoCapture 2.0 handheld dermoscope.

\section{Materials and Methods}

An observational analytical study was conducted on 150 clinically diagnosed patients of macular facial hyperpigmented skin lesions attending the out-patient department of Dermatology, Venereology and Leprosy of Chhatrapati Shivaji Subharti Hospital, Meerut, for 18 months after approval by the university. Ethical clearance was obtained from the institutional ethical committee.

This study was prospective comparative study done in both sexes, age group $>18$ years of clinically diagnosed macular facial hyper-pigmented skin lesions among 150 clinically diagnosed subjects.

\section{Inclusion criteria}

1. Patient of age 18 years and above of both sexes.

2. Patients with complaint of hyperpigmentation (localized or generalized) over face.

3. Patients willing to comply with the study.

\section{Exclusion criteria}

1. Patients under 18 years of age.

2. Patients with hypopigmentation over face.

3. Patients undergoing phototherapy for any other dermatological conditions.

4. Patients with history of malignancy were excluded.

After all purpose and the contents of the study were fully explained, written informed consent was obtained from all patients fulfilling the inclusion criteria. A detailed history was taken regarding the age, occupation, onset, course, site and duration of hyperpigmentation, history of associated symptoms, family history of hyperpigmentation, history of use of any drugs (oral or topical) specially steroid use, use of cosmetics, history and duration of sun exposure. All patients were subjected to clinical examination. Clinical photographs were taken.

\section{Results}

In our study, mean age of the patients was $31.19 \pm 11.10$ years with minimum age of 18 years and maximum 74 years. Majority (38.7\%) were 21 - 30 years old, around $33.3 \%$ of the by $31-40$ years old, $13.3 \%$ were $\leq 20$ years old, $10.0 \%$ were $41-50$ years old and only $4.7 \%$ of them were $>50$ years old. This is shown in table no.1.

Table 1: Distribution of subjects according to age group

\begin{tabular}{|c|c|c|}
\hline Age Group (Years) & Frequency (n=150) & Percentage (\%) \\
\hline$\leq 20$ & 20 & $13.3 \%$ \\
\hline $21-30$ & 58 & $38.7 \%$ \\
\hline $31-40$ & 50 & $33.3 \%$ \\
\hline $41-50$ & 15 & $10.0 \%$ \\
\hline$>50$ & 7 & $4.7 \%$ \\
\hline $\begin{array}{c}\text { Mean Age } \pm \text { SD (Min to } \\
\text { max) }\end{array}$ & $31.19 \pm 11.10(11-74)$ Years \\
\hline
\end{tabular}


In our study, more than two third of the patients (72.0\%) were females, while $28.0 \%$ were males. Males to females ratio was $7: 18$. This is shown in table no. 2 .

Table 2: Distribution of subjects according to gender

\begin{tabular}{|c|c|c|}
\hline Sex group & Frequency (n=150) & Percentage (\%) \\
\hline Male & 42 & $28.0 \%$ \\
\hline Female & 108 & $72.0 \%$ \\
\hline
\end{tabular}

In our study majority of the patients (55.3\%) were married while $44.7 \%$ patients were unmarried. This is shown in table no.3
Table 3: Distribution of subjects according to marital status

\begin{tabular}{|c|c|c|}
\hline Marital status & Frequency (n=150) & Percentage (\%) \\
\hline Married & 83 & $55.3 \%$ \\
\hline Unmarried & 67 & $44.7 \%$ \\
\hline
\end{tabular}

In our study majority of the patients (46.7\%) were suffering for 13-60 months; while 38 subjects (25.3\%) suffered for 112 months, followed by 30 subjects $(20.0 \%)$ suffered for 61 120 months, followed by only 8 subjects (5.3\%) suffered for $>120$ months; while less than one month disease duration was observed in only 4 subjects $(2.7 \%)$. This is shown in table no.4.

Table 4: Duration of disease group wise distribution of studied patients

\begin{tabular}{|c|c|c|}
\hline Duration of disease (Months) & Frequency (n=150) & Percentage (\%) \\
\hline$<1$ Month & 4 & $2.7 \%$ \\
\hline $1-12$ & 38 & $25.3 \%$ \\
\hline $13-60$ & 70 & $46.7 \%$ \\
\hline $61-120$ & 30 & $20.0 \%$ \\
\hline$>120$ & 8 & $5.3 \%$ \\
\hline Mean \pm SD (Min to max) & $45.59 \pm 39.89(1-204)$ Months \\
\hline
\end{tabular}

In our study, incomplete reticular pigmentation was the most common (35.3\%) dermoscopic finding. It was followed by telangiectasia (25.3\%), complete reticular pattern (14.0\%), diffuse pigmentation and granular pattern (12.0\%), brown globules and non-specific features (10.7\%) were other findings. This is shown in table no.5.

Table 5: Distribution of subjects according to their dermoscopic findings

\begin{tabular}{|c|c|c|}
\hline Dermoscopic Findings & Frequency (n=150) & Percentage (\%) \\
\hline Incomplete reticular pigmentation & 53 & $35.3 \%$ \\
\hline Telangiectasia & 38 & $25.3 \%$ \\
\hline Complete reticular pattern & 21 & $14.0 \%$ \\
\hline Diffuse pigmentation & 18 & $12.0 \%$ \\
\hline Granular pattern & 18 & $12.0 \%$ \\
\hline Non-specific features & 16 & $10.7 \%$ \\
\hline Brownish globules & 10 & $6.7 \%$ \\
\hline Reticuloglobular pattern & 8 & $5.3 \%$ \\
\hline Bluish grey dots & 5 & $3.3 \%$ \\
\hline Blotches & 4 & $2.7 \%$ \\
\hline Others & 15 & $10.0 \%$ \\
\hline
\end{tabular}

The most common dermoscopic pattern in melasma were incomplete reticular pigmentation (46.5\%) and telangiectasia (29.8\%) followed by complete reticular pattern (18.4\%), granular pattern (14.9\%), and non-specific features $(14.0 \%)$. In case of ochronosis majority of the cases brownish globules (50.0\%) followed by reticuloglobular pattern and diffuse pigmentation pattern in each 33.3\%, and telangiectasia in only $16.7 \%$ cases. but in case of periocular melanosis majority of the cases showed blotches (57.1\%) followed by telangiectasia and diffuse pigmentation pattern in each $28.6 \%$. This is shown in table no.6.

Table 6: Distribution of subjects on the basis of clinical diagnosis and Dermoscopic findings

\begin{tabular}{|c|c|c|c|c|}
\hline \multirow{2}{*}{ Dermoscopic Findings } & \multicolumn{3}{|c|}{ Diagnosis } \\
\cline { 2 - 5 } & Melasma (n=114) & Ochronosis (n=6) & Periocular Melanosis (n=7) & Lichen planus pigmentosus (n=4) \\
\hline Incomplete reticular pigmentation & $53(46.5 \%)$ & $0(0.0 \%)$ & $0(0.0 \%)$ & $0(0.0 \%)$ \\
\hline Telangiectasia & $34(29.8 \%)$ & $1(16.7 \%)$ & $2(28.6 \%)$ & $0(0.0 \%)$ \\
\hline Complete reticular pattern & $21(18.4 \%)$ & $0(0.0 \%)$ & $0(0.0 \%)$ & $0(0.0 \%)$ \\
\hline Granular Pattern & $17(14.9 \%)$ & $0(0.0 \%)$ & $0(0.0 \%)$ & $1(25.0 \%)$ \\
\hline Diffuse pigmentation & $4(3.5 \%)$ & $2(33.3 \%)$ & $2(28.6 \%)$ & $0(0.0 \%)$ \\
\hline Reticuloglobular Pattern & $4(3.5 \%)$ & $2(33.3 \%)$ & $0(0.0 \%)$ & $0(0.0 \%)$ \\
\hline Blotches & $0(0.0 \%)$ & $0(0.0 \%)$ & $4(57.1 \%)$ & $1(25.0 \%)$ \\
\hline Brownish Globules & $4(3.5 \%)$ & $3(50.0 \%)$ & $0(0.0 \%)$ & $1(25.0 \%)$ \\
\hline Bluish Grey Dots & $0(0.0 \%)$ & $0(0.0 \%)$ & $0(0.0 \%)$ & $0(0.0 \%)$ \\
\hline Non-specific features & $16(14.0 \%)$ & $0(0.0 \%)$ & $0(0.0 \%)$ & $3(75.0 \%)$ \\
\hline Others & $3(2.6 \%)$ & $0(0.0 \%)$ & $2(28.6 \%)$ & \\
\hline
\end{tabular}

\section{Discussion}

The skin in humans presents in a variety of shades depending on various factors like race, climate, light, ethnicity and race, anatomical site and lifestyle of the individual. A great geographical (Climatic) variation observed in India. Indian skin shows great variability in skin 
color and other specific features as India is home to a variety of ethnicities ${ }^{[9]}$. A study from four cities of India demonstrated that facial skin color heterogeneity may be present in as high as $80 \%$ of people, regardless of age or sex [10]. These factors have a profound effect on the quality of life and causes emotional distress to the sufferers [11]. Disorders causing hyperpigmentation are more common in India than disorders causing hypopigmentation [12]. diagnosing skin conditions accurately and reducing the psychosocial burden on patients. Even a small discolored area over face can cause huge mental distress. Obsession of Indian society with fair skin makes one with facial hyperpigmentation feel under confident and dejected socially.

This study was, therefore, conducted to describe dermoscopic features of common macular facial hyperpigmented skin lesions. The study provides insight about the diagnosis and treatment of common macular facial hyperpigmented skin lesions.

The present observational study was conducted in patients of macular facial hyperpigmented skin lesions attending the out-patient department of Dermatology, Venereology and Leprosy of Chhatrapati Shivaji Subharti Hospital, Meerut, UP. After approval from the university and obtaining Ethical clearance from the institutional ethical committee; a total of 150 clinically diagnosed macular facial hyperpigmented skin lesions adult either sex patients attending OPD in study duration of 18 months were included in this study. Details of patients such as socio-demographic profile, duration of disease, photosensitivity, and treatment history, clinical diagnosis of disease, area involvement and dermoscopic finding was collected. This methodology was used in several studies. Kaur S et al. ${ }^{[13]}$ conducted a prospective observational cohort study of the clinicodermatoscopic and histopathological evaluation of cervicofacial hypermelanosis and concluded that the hyperpigmentary disorders are great mimickers. Clinical examination alone can misdiagnose certain conditions. Dermatoscope, a novel office tool, when used along with time tested modality like histopathology, can reduce the diagnostic burden of clinicians while treating hyperpigmentary conditions. Adil $\mathrm{M}$ et al. ${ }^{[14]}$ performed a retrospective; hospital based descriptive study in a tertiary care hospital of North India of the hyper-pigmented skin conditions and concluded that the melasma was the leading cause of hyperpigmentation. Post inflammatory hyperpigmentation and lichen planus pigmentosus are important causes of pigmentation in colored skin, unlike in fair skinned individuals. Sandhu S et al. ${ }^{[15]}$ studied the overview of the dermoscopy in disorders of hyperpigmentation affecting individuals with skin of color and concluded that the dermoscopy is useful in diagnosis, prognosis, and follow up of disorders of hyperpigmentation. With regular practice and attention to detail towards dermoscopic findings, it can be a useful tool in dermatology practice in general and for disorders of hyperpigmentation.
In our study, more than two third of the patients $(72.0 \%)$ were females, the other $28.0 \%$ were males. Males to female ratio were 1:2.6. Melasma is more common in women Sarkar et al. ${ }^{[9]}$ conducted an etiological and histological study in Indian males with melasma and found that men represent 20.5 - 25.83\% of the cases. Kavya M \& Nataraj $\mathrm{HV}^{[16]}$ reported the female to male was 1:1.8 in their study. While Shahana et al. ${ }^{[17]}$ reported female preponderance in facial hyperpigmentation where female to male ratio was $4: 1$. Kaur S et al. ${ }^{[13]}$ also reported a ratio of $4: 1$ in their study. The higher frequency of female cases can be attributed to the reason that females are more concerned about their cosmetic appearance as compared to the males. Hence more number of females seeks medical advice and present to the dermatology department.

In present study we observed that the prevalence of diagnosis like periocular melanosis $(4.7 \%)$, ochronosis (4.0\%), lichen planus pigmentosus (2.7\%), seborrheic keratosis $(2.0 \%)$, nevus of Ota $(2.0 \%)$, post inflammatory hyperpigmentation (1.3\%), fixed drug eruption (1.3\%), Riehl's melanosis (1.3\%) and other findings (facial dyschromia, perioral hyperpigmentation, Darier's disease, lentigines) were $4.7 \%$. In a similar study Adil $\mathrm{M}$ et al. ${ }^{[14]}$ reported the Lichen planus pigmentosus (7.4\%), fixed drug eruption (1.5\%), post inflammatory hyperpigmentation (12.5\%), and peri-orbital melanosis (4.5\%). Kavya M \& Nataraj HV [16] reported the post inflammatory hyperpigmentation (25\%) and periocular hypermelanosis (12\%). Other causes were Riehl's melanoses (6\%), ephelides and drug induced hypermelanosis 5\% each, LPP (4\%), perioral hypermelanosis (3\%) \& ashy dermatoses (2\%). Kaur S et al. ${ }^{[13]}$ reported the Lichen planus pigmentosus (LPP) (23.0\%), post-inflammatory hyperpigmentation (PIH) (7.0\%), periorbital hyperpigmentation $(\mathrm{POH})(6.0 \%)$, Riehls melanosis (RM) $(2.0 \%)$. The variation in percentage of type of facial hyperpigmented skin lesions reason was the post inflammatory hyperpigmentation was acquired pigmentary disease produced due to end result of inflammation in diseases like acne, psoriasis, lichen planus, eczematous dermatitis and insect bites. Coloured skin is more likely to develop post inflammatory pigmentation, that too with greater severity ${ }^{[18]}$. LPP in our study could be explained on the basis of almost universal practice of using the above mustard oils on scalp in rural Western Uttar Pradesh, where a large number of our patients hail from. This variability may be explained on the selection criteria of the patients. The limitations were this was an observational study with data for a small number of patients and limited study duration of 18 months. 


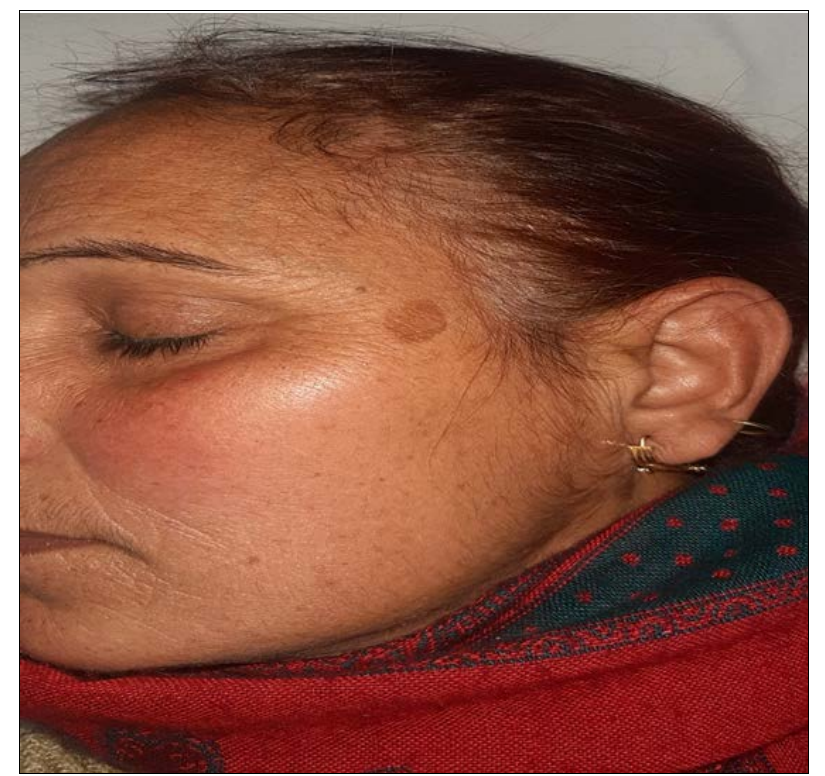

Fig 1: Clinical picture of seborrheic keratosis (flat variant)

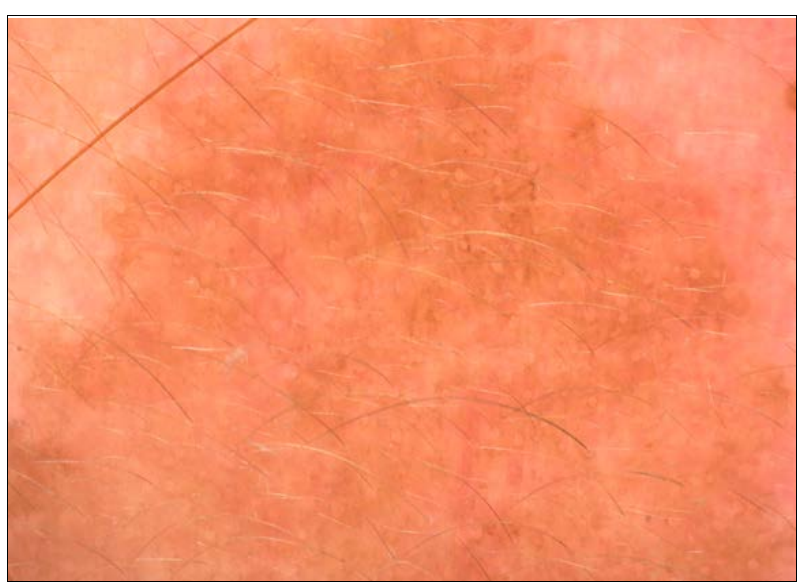

Fig 2: Dermoscopy of seborrheic keratosis (flat variant) revealing heterogeneous pigmentary network with moth eaten border and telangiectasia.

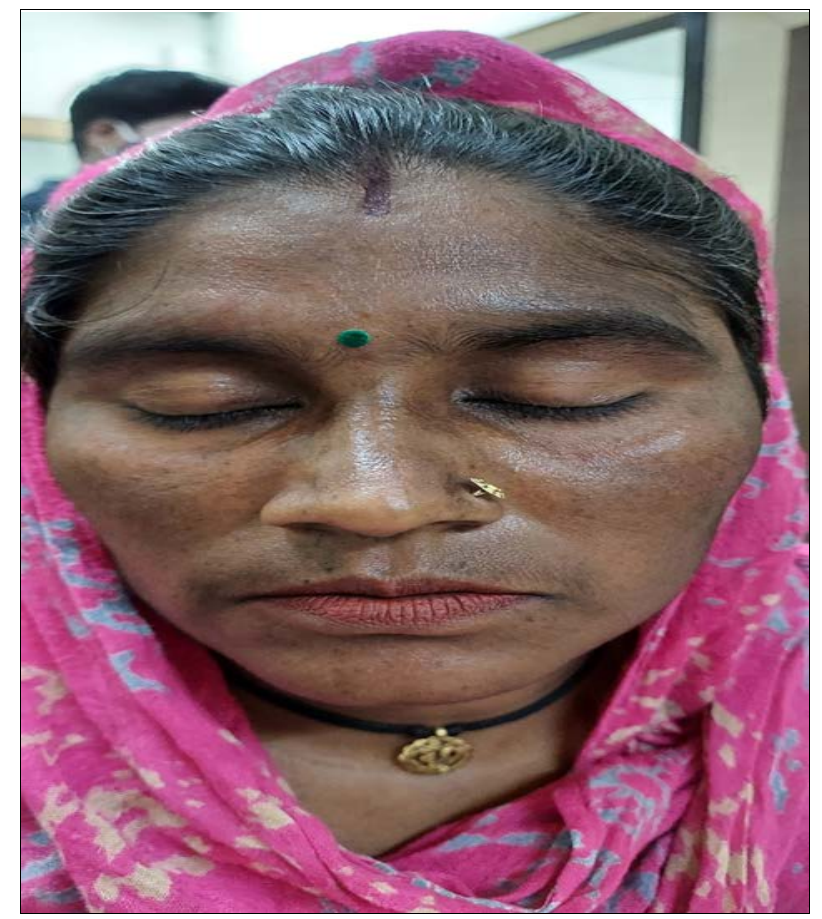

Fig 3: Clinical picture of erythema dyschromicum perstans (ashy dermatosis of Ramirez).

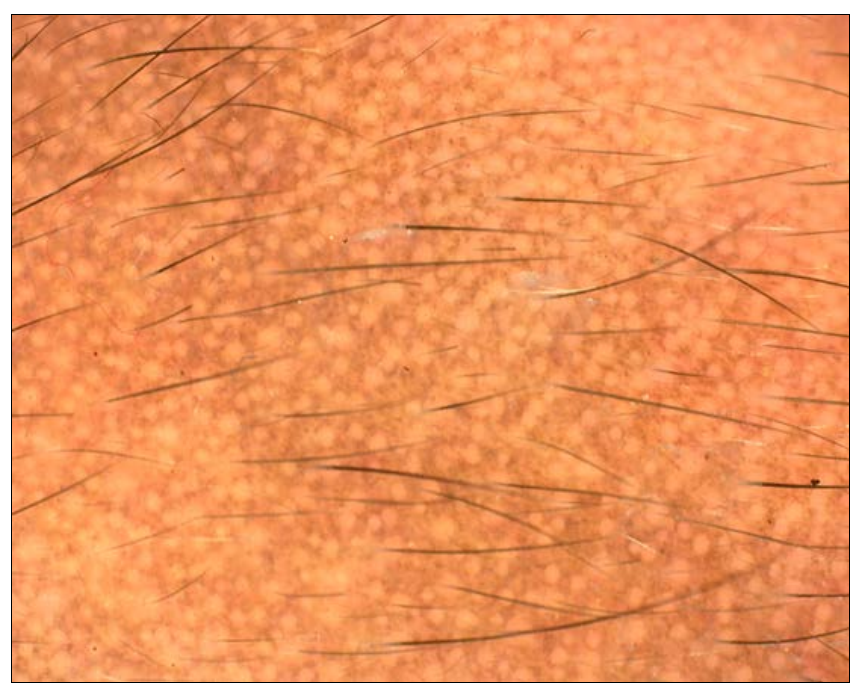

Fig 4: Dermoscopy of erythema dyschromicum perstans showing complete reticular pattern pigmentation at some places and at other areas the pigmentation are more discrete speckled and granular.

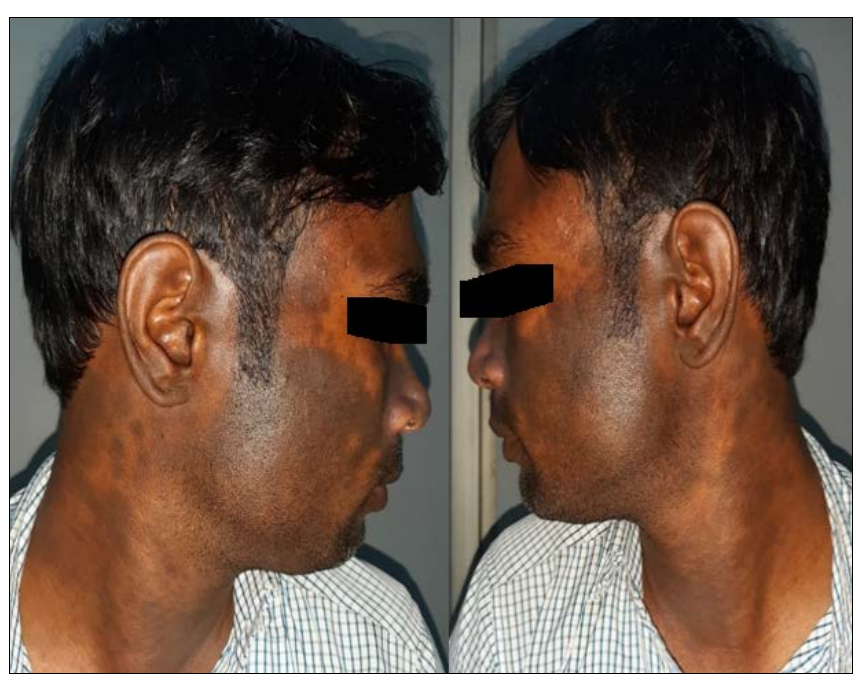

Fig 5: Clinical picture of lichen planus pigmentosus

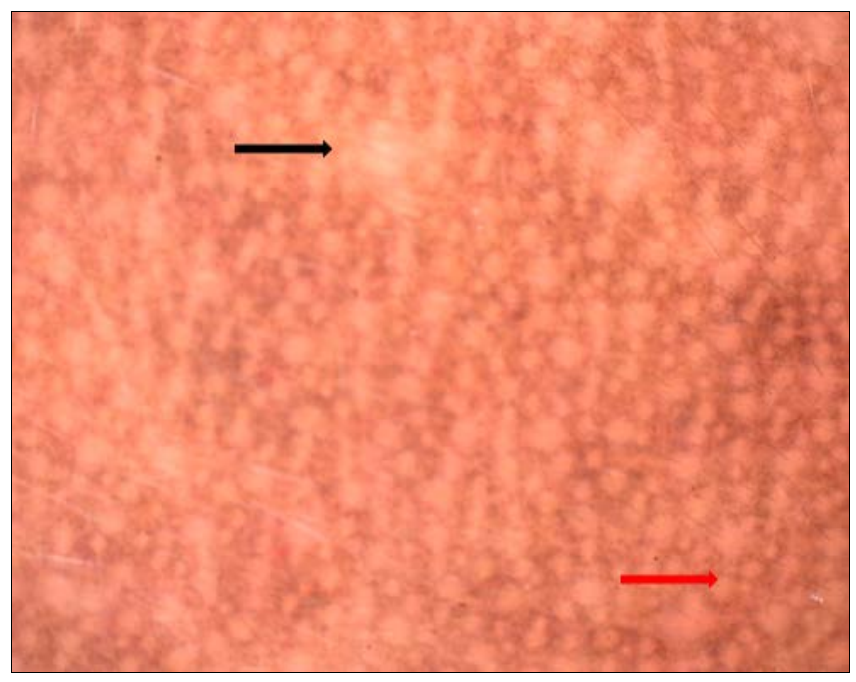

Fig 6: Dermoscopy of lichen planus pigmentosus revealing non uniform pigment accentuation in a reticular pattern, at places quantity of pigment appears to be more (red arrow). Other places it appears to be less (black arrow).

\section{Conclusion}

In conclusion, facial hypermelanosis is a clinical feature of a 
diverse group of disorders most common in middle aged females exposed to sunlight having genetic predisposition. Melasma is the major contributor to the load of patients with hyperpigmentation. Post inflammatory hyperpigmentation is fairly common in skin type IV-VI and occupies the second spot.

Diagnosis is generally based on detailed personal, family history and clinical features aided with relevant investigations depending on individual case. Dermoscopy is useful in diagnosis, prognosis, and follow up of disorders of hyperpigmentation. With regular practice and attention to detail towards dermoscopic findings, it can be a useful tool in dermatology practice in general and for disorders of hyperpigmentation.

\section{References}

1. Anstey AV. Disorders of Skin Colour. In: Rooks textbook of dermatology. 8th edition. 58, 1-58.59.

2. Duma S. Dermoscopy of pigmented skin lesions. In: European Handbook of Dermatological Treatments, $3^{\text {rd }}$ E Republic of Macedonia. 2015;1167-77.

3. Sharma VK, Sahni K, Wadhwani AR. Photodermatoses in pig-mented skin. Photochem Photobiol Sci. 2013;12:65-77.

4. Wakamatsu K, Kavanagh R, Kadekaro AL, et al. Diversity of pig-mentation in cultured human melanocytes is due to differences in the type as well as quantity of melanin. Pigment Cell Res. 2006;19:154-62.

5. Vashi NA, Kundu RV. Facial hyperpigmentation: causes and treatment. British Journal of Dermatology. 2013;169(3):41-56.

6. Deinlein T, Zalaudek I. Hyperpigmentation [Internet]. Rigopoulos D, Katoulis AC, editors. Hyperpigmentation. CRC Press; 2017. Available from: https://www/taylorfrancis.com/books.9781351668323.

7. Khanna N, Rasool S. Facial melanoses: Indian perspective. Indian $\mathrm{J}$ Dermatol Venereol Leprol. 2011;77:552-63.

8. Mutalik SD, Pethe SV, Nikam BP, Rasal YD. Facial frictional melanosis in Indian patients: Defining the entity. Clin Dermatol Rev. 2019;3:78-83.

9. Jaswal IJ. Pigmentary variation in Indian populations. Acta Anthropogenet. 1983;7:75-83.

10. Hourblin V, Nouveau S, Roy N, de Lacharrière O. Skin complexion and pigmentary disorders in facial skin of 1204 women in 4 Indian cities. Indian J Dermatol Venereol Leprol. 2014;80:395-401.

11. Ortonne JP, Passeron T, Srinivas C. In: Pigmentary Disorders, Prevention, Treatment and Cosmetics Contributions. In: Srinivas C, Verschoore M, editors. Basic Science for Modern Cosmetic Dermatology. New Delhi: Jaypee Brothers Medical Publishers, 2015, 7590.

12. Dogra S, Sarangal R. Pigmentary disorders: An insight, Pigment International. 2014;1:5-7.

13. Kaur S, Kaur J, Sharma S. Clinico-dermatoscopic and histopathological evaluation of cervico-facial hypermelanosis: A study from a tertiary care hospital. Pigment Int. 2020;7:87-95.

14. Mohammad Adil, Amin SS, Arif T, Dorjay K, Dinesh Raj R, Bansal R. Hyperpigmented Skin Conditions: A Study of Pattern and Prevalence From A Tertiary Care Hospital Of North India, International Journal of Current Advanced Research. 2017;06(04):3562-3565.
15. Sandhu S, Neema S, Radhakrishnan S. Dermoscopy of disorders of hyperpigmentation. Pigment Int. 2021;8:14-24.

16. Kavya M, Nataraj HV. Clinico-epidemiological study of facial hypermelanoses. Sch. J App Med Sci. 2014;2:1621-26.

17. Shahana M, Padma A, Khan PM. Study of clinical patterns of facial pigmentation. Int $\mathrm{J}$ Biomed Res. 2016;7:94-8. 